\title{
¿LOS FUTUROS DOCENTES ESTÁN COMPROMETIDOS CON LA JUSTICIA SOCIAL? VALIDACIÓN Y APLICACIÓN DE LA TRADUCCIÓN AL CASTELLANO DE LA ESCALA DE JUSTICIA SOCIAL
}

\author{
Are future teachers committed to social justice? Validation and application \\ of the Spanish translation of Social Justice Scale
}

\author{
NINA HIDALGO*1 \\ CYNTHIA MARTÍNEZ-GARRIDO**2 \\ HAYLEN PERINES ${ }^{\star * * 3}$ \\ *Universidad Autónoma de Madrid. España. Correo: nina.hidalgo@uam.es \\ **Universidad Autónoma de Madrid. España. Correo: cynthia.martinez@uam.es \\ ***Universidad de La Serena. Chile. Correo: haylen.perines@userena.cl
}

Recibido: 24-02- 2017

Revisado: 26-04-2017

Aceptado: 16-06-2017

Resumen: Esta investigación tiene un doble propósito: por un lado, traducir al castellano la Escala de Justicia Social de Torres-Harding, Siers y Olson (2012) y validar el instrumento; por otro, conocer las actitudes de los futuros maestros hacia la justicia social y su compromiso con ella. Luego de traducir y validar la escala, se realizó un estudio descriptivo ex post facto con 326 estudiantes de magisterio en Educación Infantil y Primaria de la Universidad Autónoma de Madrid (España). Los resultados del estudio muestran que los maestros en formación de Primaria tienen una mejor actitud hacia la justicia social que los futuros maestros de Infantil; y que las mujeres tienen una mejor actitud que los hombres. La escala traducida es una valiosa herramienta para investigadores castellanohablantes que busquen identificar actitudes relacionadas con la justicia social.

Palabras clave: justicia social; escala; validación; castellano; actitudes.

\begin{abstract}
This research has a double purpose: on the one hand, to translate into Spanish the Social Justice Scale of Torres-Harding, Siers and Olson (2012) and to validate the instrument; on the other hand, to know the attitudes and commitment of future teachers towards social justice. After translating and validating the scale, an ex post facto descriptive study was carried out with 326 undergraduate teacher training students at the Universidad Autónoma de Madrid (Spain). The results of the study show that Primary School teachers have a better attitude towards social justice than future Kindergarten teachers, and that women have a better attitude than men. The translated scale is a valuable tool for Spanish-speaking researchers seeking to identify attitudes related to social justice.
\end{abstract}

Keywords: social justice; scale; validation; Spanish; attitudes.

\footnotetext{
${ }^{1}$ Profesora en la Universidad Autónoma de Madrid. Doctora en Educación en la Universidad Autónoma de Madrid (España). Miembro del grupo de investigación Cambio Educativo para la Justicia Social y de la Red sobre Liderazgo y Mejora Educativa (RILME).

${ }^{2}$ Profesora en la Universidad Autónoma de Madrid (España). Doctora en Ciencias de la Educación, Universidad Autónoma de Madrid (España). Miembro del grupo de investigación Cambio Educativo para la Justicia Social y de la Red sobre Liderazgo y Mejora Educativa (RILME).

${ }^{3}$ Académica e Investigadora del Instituto interdisciplinario de Ciencia y Tecnología, Universidad de La Serena (Chile). Doctora en Educación de la Universidad Autónoma de Madrid (España). Máster en Gestión Educativa (UMCE, Chile), Profesora de Castellano y Filosofía (ULS, Chile).
} 


\section{INTRODUCCIÓN}

En la actualidad vivimos en una sociedad desigual donde no todas las personas pueden acceder a las mismas oportunidades, beneficios o posibilidades. Esta injusticia unida a desigualdades de género, clase social, cultura, capacidad, orientación sexual, lengua, entre otras, genera una preocupante exclusión de aquellos que forman parte de los grupos sociales más desfavorecidos (Murillo y Hernández-Castilla, 2011a). Frente a estas injusticias el término justicia social adquiere más relevancia que nunca, tanto es así que el filósofo Julián Marías ya en 1974 afirmó que "el siglo XX no sería entendible sin ese término" (p.7). En palabras de Murillo y Hernández-Castilla (2011b) "el anhelo por una mayor justicia social surge, en primer lugar, por la nítida percepción de las múltiples y crecientes injusticias que nos rodean; pero también en la búsqueda de una mejor sociedad" (p.8).

En este contexto, la educación es, quizá, uno de los mecanismos más valiosos que se tienen a disposición para lograr que la sociedad sea cada vez más justa. Aunque es cierto que la educación por sí sola difícilmente puede cambiar la sociedad, sin ella es imposible hacerlo. Coincidimos con Adams, Bell y Griffin (2007) en considerar que la Educación para la justicia social tiene como finalidad conseguir una sociedad más justa. En el marco teórico se profundizará en ello, como también en las implicancias de su definición.

Una de las líneas de investigación que surgen a partir del concepto de justicia social tiene que ver con la forma concreta de medir este término a través de escalas. Gran parte de ellas lo realizan en términos generales, como Corning y Myers (2002) o bien haciendo referencia a aspectos específicos como la participación política (Caprara, Vecchione, Capanna y Mebane, 2009), la equidad (Colquitt, 2001; Rasinski, 1987) o la salud mental (Dean, 2009; Ritchhart, 2002).

Existe una escala centrada en un aspecto más específico: la Escala de Justicia Social (Social Justice Scale, SJS) de Torres-Harding, Siers y Olson (2012), quienes pretenden evaluar las actitudes y los valores relacionados a la justicia social, así como las intenciones de participar en actividades relacionadas con ella. $Y$ esta escala es precisamente la base del presente estudio, que tiene un doble objetivo. En primer lugar, validar una versión de la escala traducida al castellano y, en segundo lugar, conocer las actitudes hacia la justicia social de los futuros maestros ${ }^{4}$.

\footnotetext{
${ }^{4}$ Resultados parciales de este estudio fueron presentados en un congreso internacional debido a su interés para la comunidad científica (Martínez-Garrido y Perines, 2015).
} 


\section{MARCO TEÓRICO}

Con el fin de conocer qué principios teóricos sustentan la escala, el marco teórico se estructura a través de una breve explicación de la escala y sus fundamentos teóricos y conceptuales.

\section{La Escala de Justicia Social (SJS)}

La SJS 5 es un instrumento conformado por 24 ítems valorados en una escala tipo Likert de 1 (muy en desacuerdo) a 7 (muy de acuerdo), que evalúa cuatro componentes (subescalas) del compromiso de las personas hacia la justicia social (Torres-Harding et al., 2012):

- Actitudes generales hacia la justicia social

- Percepción de control del comportamiento al participar en actividades de justicia social

- Normas sociales percibidas respecto de la justicia social

- Intenciones de participar en el trabajo de la justicia social o activismo.

Existen numerosas investigaciones que han utilizado la SJS. Por un lado, los propios autores la utilizan para investigaciones complementarias sobre los valores y la religiosidad como predictores del compromiso con la justicia social y el sentido de comunidad (Torres-Harding, Carollo, Schamberger y Clifton-Soderstrom, 2013). Por otro, también se utiliza en investigaciones centradas en la justicia social al interior de las universidades, específicamente en la misión de la Universidad como predictores de la justicia social (Torres-Harding, Diaz, Schamberger y Carollo, 2015).

Por otro lado, estudios como los de Poteat, Scheer, Marx, Calzo y Yoshikawa (2015) o Kozlowski, Ferrari y Odahl (2014) fundamentan sus investigaciones en la SJS, dada la fiabilidad y consistencia que presentan en su estudio original. Esta fiabilidad y consistencia la convierten en un instrumento adecuado para medir las actitudes hacia la justicia social. Asimismo, Fabian (2012) y Kozlowski et al. (2015) también usan en sus investigaciones el concepto de justicia social en el que se fundamenta dicha escala.

En un análisis de distintas escalas realizado por Fietzer y Ponterotto (2015) se lleva a cabo una revisión de los instrumentos psicométricos existentes para promover actitudes hacia la justicia social. Los autores afirman que la SJS es uno de los pocos instrumentos que ofrece evidencia clara de las actitudes hacia la justicia social sin variaciones por poblaciones, siendo una herramienta útil para conocer cuáles son las actitudes hacia la justicia social de distintas personas.

\footnotetext{
${ }^{5}$ La escala puede encontrarse en la siguiente publicación: https://www.ncbi.nlm.nih.gov/pubmed/22080396
} 


\title{
Fundamentación teórica de la Escala de Justicia Social
}

\author{
Escalas que fundamentan la SJS
}

Torres-Harding et al. (2012) se apoyan en cuatro instrumentos de justicia social para evaluar la validez convergente y discriminante de las cuatro subescalas encontradas. Estos instrumentos son:

- Escala de creencia global en un mundo justo (Lipkus, 1991): compuesta por 7 ítems. Mide la creencia en un mundo justo como un proceso de atribución donde las personas reciben en la vida lo que se merecen, y en donde, por lo general, cada individuo es responsable de su propia buena fortuna o desgracia. El rango de puntuaciones fluctúa entre 7 y 42.

- Escala de motivación por el servicio público de Perry - Versión abreviada (Coursey y Pandey, 2007): compuesta por 10 ítems derivados de la escala original de Perry (1996). Esta escala abreviada incluye 3 ítems de la subescala de la política, 4 elementos de la subescala deber cívico (vocación de servicio público) y 3 artículos de la subescala de compasión. Esta escala Intenta medir el grado en que los individuos están motivados para trabajar en el servicio público.

- Escala de racismo simbólico (Henry y Sears, 2002): compuesta por 8ítems medidos de 1 a 4 , cuya suma proporciona la puntuación total de la escala. Mide formas sutiles de racismo expresadas contra los afroamericanos.

- Escala de neosexismo (Tougas, Brown, Beaton y Joly, 1995): compuesta por 11 ítems en una escala de 1 a 7 . Mide el grado en que un participante apoya creencias neosexistas. Estas creencias se definen en la medida en que se niega la existencia de las desigualdades de género, mientras que de manera simultánea se culpa a las mujeres.

\section{El concepto de justicia social}

El concepto de justicia social puede entenderse desde diferentes perspectivas, ya que es un término dinámico que depende de la sociedad en la que se inserta (Griffiths, 2003). De los múltiples conceptos que existen de justicia social, Torres-Harding et al. (2012) construyen los ítems de su SJS basándose principalmente en las propuestas de Constantine, Hage, Kindaichi y Bryant (2007); Fouad, Gerstein y Toporek (2006) y Prilleltensky (2001).

Constantine et al. (2007) definen a la justicia social de la siguiente manera:

La justicia social refleja una valoración fundamental de la equidad en la participación de todos los ciudadanos y un reparto equitativo de los recursos, derechos y el trato de las personas marginadas y grupos de personas que no comparten el mismo poder en la sociedad debido a su raza, etnia, edad, nivel socioeconómico, patrimonio religioso, la capacidad física, la orientación sexual o su posición o estatus (p. 24). 
Por otro lado, Fouad et al. (2006) describen la justicia social como el "principio que vela porque las oportunidades y los recursos se distribuyan equitativamente dentro de la sociedad, y que los individuos y grupos de trabajo garanticen la equidad, cuando estos recursos no son correctamente distribuidos" (p.13). Por último, en el trabajo desarrollado por Prilleltensky (2001) se describe la justicia social como:

La promoción de la justicia y la asignación equitativa de los poderes de negociación, recursos y las obligaciones de la sociedad considerando en el poder diferencial las necesidades y las habilidades para expresar sus deseos de los ciudadanos (p.754).

Para Torres-Harding et al. (2012) hablar de justicia social es considerar que las desigualdades estructurales y sociales deberían ser reducidas al mínimo; y que la sociedad debe trabajar para promover el empoderamiento de aquellos que sufren las desventajas sociales. Otras formas de definir justicia social, como las propuestas por Fraser (2008) o Murillo y Hernández-Castilla (2011), consideran que ésta se estructura a partir de tres dimensiones complementarias: la redistribución, entendida como una distribución equitativa de los bienes primarios en la sociedad (Rawls, 1971); el Reconocimiento, relativa a la ausencia de dominación cultural y valoración de las características de todas las personas (Honneth, 2003); y la representación o participación, definida por la participación activa de las personas en la sociedad, especialmente aquellas que han sido tradicionalmente oprimidas (Fraser, 2008; Young, 2000). Desde la perspectiva de Torres-Harding et al. (2012) la participación, la colaboración y el empoderamiento de los ciudadanos son ejes imprescindibles sobre los que se construye el término de justicia social en la escala que desarrollan. De acuerdo con la perspectiva tridimensional presentada, en la SJS es asumida principalmente desde una concepción de redistribución, relativa a la distribución de los recursos en la sociedad. El poder de una distribución equitativa de los recursos está en manos de las instituciones que componen la sociedad, las que, según autores como Rawls (1971), si no son justas deben ser abolidas.

No obstante, de acuerdo a la perspectiva tridimensional presentada, también se encuentran otras formas de concebir la justicia social presentes en menor medida en la escala SJS, más cercanas a concepciones de reconocimiento, esto es, considerando que una sociedad es realmente justa cuando reconoce y valora a todos sus ciudadanos, especialmente a aquellos que forman parte de grupos marginados — por raza, etnia, cultura, orientación sexual, capacidad- (Fraser, 2006; Fraser y Honneth, 2006; Honneth, 2007; Young, 2007, 2011). Para Honneth (2007) el reconocimiento es el valor supremo de la justicia social, siendo la dimensión más relevante para lograr una sociedad más justa. Para este autor, uno de los más influyentes de la escuela de Frankfurt, el reconocimiento permite valorar la dignidad de todos los seres humanos y es el eje rector de la sociedad moderna, y cualquier otro principio debe quedar supeditado a este. La tercera dimensión de la justicia social que no se ve reflejada en la escala SJS es la 
representación, en otras palabras, la participación crítica y activa de los ciudadanos en la sociedad y los estamentos de poder y decisión (Fraser, 2008; Young, 2007, 2011).

La justicia social en la acción de la escala

Tal y como señalan Cook (1990) y Fox (2003) el verdadero cambio hacia la justicia social está relacionado con la puesta en marcha de acciones y prácticas tangibles. En este sentido, es fundamental conocer las actitudes de las personas, hacerlas conscientes de ellas y ver cómo estas influyen en sus acciones diarias, especialmente aquellas relacionadas con la consecución de una sociedad más justa. Así, para comprender qué es justicia social es importante saber cómo las personas e instituciones traducen este concepto a su acción o práctica cotidianas.

Siguiendo la idea de Fouad et al. (2006), la promoción de la justicia social implica un cambio real de las instituciones, políticas, gobiernos y estructuras económicas que perpetúan prácticas perjudiciales e injustas limitando el acceso a los recursos de la sociedad. De la misma forma, la práctica de la justicia social puede observarse en el cambio de las regularidades de un sistema, es decir, en la manera en que se hacen típicamente las cosas en las instituciones sociales. Una práctica socialmente justa requiere la participación, el respeto y el reconocimiento de todas las personas, de tal forma que las instituciones promuevan iniciativas que empoderen a todos y cada uno de los ciudadanos (Constantine et al., 2007; Fouad et al. 2006; Goodman et al., 2004; Prilleltensky y Nelson, 2002). Desde una perspectiva más educativa, la aceptación de las dimensiones de justicia social (redistribución, reconocimiento o representación) supone un paso crítico para promover la participación de los estudiantes en la acción social. De nuevo el salto desde el cambio de actitudes a la puesta en práctica de acciones resulta imprescindible para realmente concebir la justicia social, en este caso, en la escuela (Goodman, 2000).

Dadas las dificultades que conlleva la medición de la justicia social, TorresHarding, Siers y Olson se sirven del modelo cognitivo social elaborado por Ajzen (1991) denominado Modelo de Comportamiento Planificado. En él, el autor analiza cómo las actitudes y los constructos enlazados entre sí predicen los comportamientos (acciones) sensibles a la justicia social de los individuos. De acuerdo con Cook (1990) y Fox (2003), solo cuando estas acciones son llevadas a cambio se puede producir un cambio efectivo hacia la justicia social. El principio central de la teoría de Ajzen (1991) se basa en que la mejor manera de predecir la acción conductual de un individuo es conociendo cuál es la intención explícita que este tiene para actuar (figura 1). A su vez, señala el autor, las intenciones de actuación se predicen por las actitudes que el propio individuo tiene hacia la acción, las normas subjetivas en torno a la acción y el control del comportamiento percibido orientado ella. La escala de Torres-Harding et al. (2012) es la primera que incorpora el modelo Ajzen para medir las variables que predicen acciones que persiguen una sociedad más justa. 
Figura 1. Modelo del Comportamiento Planificado de Ajzen (1991)

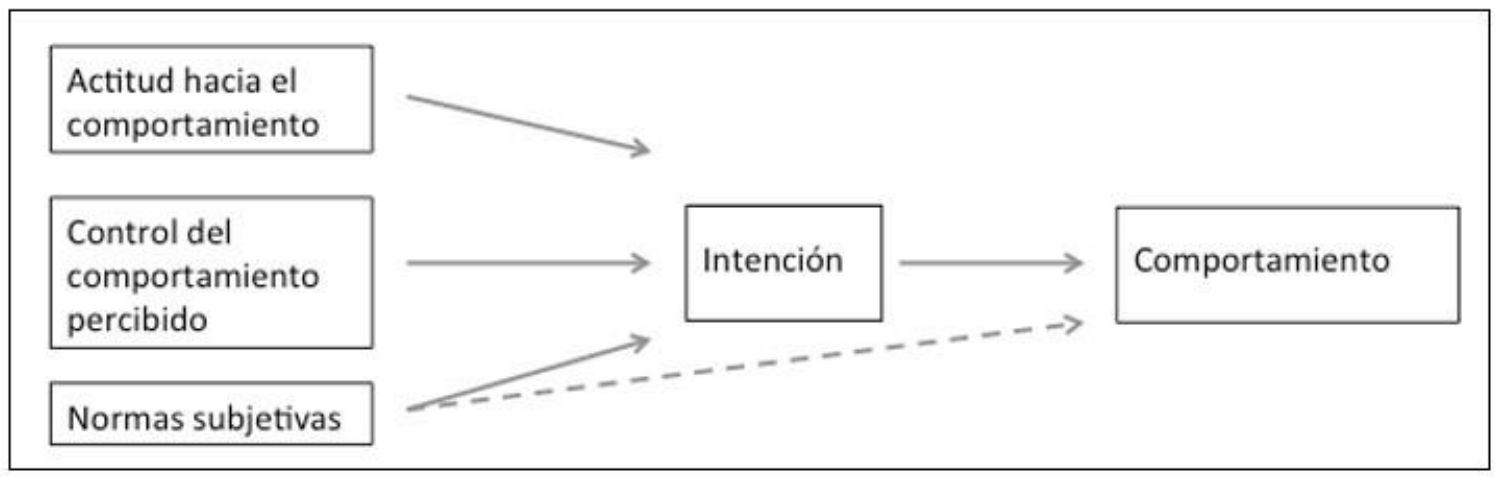

Fuente: Ajzen (1991, p. 182)

Los componentes del modelo de Ajzen (1991, p.182) se definen como:

- Actitud hacia el comportamiento: disposición para hacer un determinado comportamiento. Una actitud orientada a la justicia social implicaría la aceptación de un individuo de los ideales de justicia social y valores relacionados con ella, como la creencia de que se debe actuar para fomentarla. En este modelo, las actitudes de cada sujeto serían un importante predictor de las intenciones y las prácticas orientadas a la justicia social desarrolladas por las personas.

- Control del comportamiento percibido: capacidad percibida para realizar un acto. En un contexto de justicia social esto puede implicar la medida en que una persona considera que es posible "hacer una diferencia", o la autoevaluación de si uno mismo puede tener un impacto en las condiciones sociales ya existentes. Ajzen (1991) señala que el control de la percepción conductual podría predecir directamente las intenciones de actuar, y, en algunos casos, también podría predecir directamente el comportamiento si la acción en sí es particularmente difícil o desafiante.

- Normas subjetivas: la inclusión de las normas subjetivas genera un mecanismo que permite que el contexto social pueda influir en la aparición de un comportamiento, de manera que las normas subjetivas también predicen las intenciones de la acción.

La SJS elaborada por Torres-Harding et al. (2012) aplica el Modelo de Comportamiento Planificado de Ajzen (1991) a las acciones de justicia social para conocer las relaciones entre las normas subjetivas y las intenciones que conducen a unas determinadas acciones orientadas a la consecución de una sociedad más justa. 


\section{MÉTODO}

\section{Objetivos y fases del estudio}

Los objetivos del presente estudio son:

- Validar la traducción de la Escala de Justicia Social de Torres-Harding et al. (2012) en el contexto español.

- Determinar cuáles son las actitudes y compromiso que los futuros docentes tienen acerca de la justicia social.

Para lograrlos se ha realizado un estudio en dos etapas: una primera fase de traducción y validación, y una segunda fase que consistió en un estudio ex post facto descriptivo.

\section{Participantes}

Para la primera fase del estudio los participantes han sido 10 expertos en traducción con reconocido dominio en el estudio de la justicia social a partir de sus publicaciones realizadas en los últimos cinco años y su vinculación con la investigación sobre Educación para la justicia social. En la segunda fase, la muestra se ha compuesto por 326 estudiantes de Magisterio en Educación Infantil y Primaria de la Universidad Autónoma de Madrid (España). La selección de la muestra responde al criterio de intencionalidad de acuerdo con los objetivos del trabajo.

\section{Propiedades psicométricas de la Escala de Justicia Social original}

En su primera versión, esta escala estuvo compuesta por 44 ítems que dan respuesta a una definición de justicia social que considera:

- El empoderamiento de las personas pertenecientes a grupos desfavorecidos

- La necesidad de minimizar las diferencias de poder y trabajar en colaboración con otros

- La ayuda a las personas para que accedan a los recursos de la comunidad o sociedad

- La necesidad de reconocer las desigualdades sociales que pueden ser perjudiciales

- La importancia de hacer una sociedad más justa para todas las personas

- La creencia de que se debe trabajar por la justicia social

Tras la creación de los 44 ítems originales, los autores utilizaron un panel de 115 sujetos (psicólogos y estudiantes de postgrado) para revisar la escala y evaluar su validez, la pertinencia de sus objetivos y su coherencia con el Modelo de Comportamiento Planificado propuesto por Ajzen (1991). 
A los 44 ítems hay que añadir cinco preguntas más que responden a condiciones demográficas de los encuestados: edad, raza, género y discapacidad (Torres-Harding, Siers y Olson, 2012, p.82). Un último ítem pregunta explícitamente si se ha hecho algo para trabajar por la justicia social e indicar si se autoidentifican como "activistas".

Los 44 ítems se contestan en una escala tipo Likert de siete alternativas que abarca desde "1: totalmente en desacuerdo" hasta "7: muy de acuerdo". Tras el análisis factorial de los resultados de la revisión, los autores de la SJS diferencian cuatro subescalas:

- Subescala 1: Actitudes hacia la justicia social: compuesta por una veintena de ítems que evalúan las actitudes, valores sociales y comportamientos relacionados con la justicia social. Un ejemplo de estos ítems es la afirmación: "Creo que es importante actuar para la justicia social".

- Subescala 2: Control del comportamiento percibido: compuesta por 14 ítems que evalúan el control del comportamiento percibido haciendo específicamente referencia a los objetivos de justicia social relacionados con la autoeficacia. Por ejemplo: "Confío en que puedo tener un impacto positivo en la vida de otras personas".

- Subescala 3: Normas subjetivas: compuesta por 6ítems que sirven para evaluar si las personas, considerando su propio contexto social, apoyan o desalientan la participación de los individuos en actividades relacionadas con la justicia social. Un ejemplo de ítem es: "Las personas que me rodean están comprometidas en actividades que abordan las injusticias sociales".

- Subescala 4: Intenciones de comportamiento: compuesta por 4ítems que examinan las intenciones de comportamiento para participar en el futuro en la acción social o en actividades relacionadas con la justicia social. Un ejemplo: "En el futuro haré todo lo que pueda por asegurarme de que todos los individuos y grupos tengan la oportunidad de hablar y ser escuchados".

\section{Análisis preliminares de la Escala de Justicia Social}

Los 44 ítems originales fueron analizados de acuerdo con los siguientes criterios: consistencia interna, análisis factorial confirmatorio, revisiones de la escala, análisis factoriales confirmatorios con modelo revisado y validez convergente y discriminante.

\section{a) Consistencia interna}

La fiabilidad y la estructura factorial de la escala se detallan a continuación. El alfa de Cronbach obtenido para la escala de 44 ítems es $\alpha=0,93$. La fiabilidad de cada subescala es (a) Actitudes $\alpha=0,89$, (b) Normas subjetivas $\alpha=0,85$, (c) Control del comportamiento 
percibido $\alpha=0,77, y$ (d) las Intenciones $\alpha=0,86$. Las subescalas obtuvieron correlaciones moderadas, de entre $p=0,29$ (entre Intenciones de comportamiento y Normas subjetivas) y $\mathrm{p}=0,56$ (entre Actitudes y Control del comportamiento percibido). Las correlaciones totales del ítem corregido variaron fluctuando entre 0,18 y 0,69 (Torres-Harding, Siers y Olson, 2012, p.83).

La adecuación de la muestra y el grado de asociación entre las variables se valoró mediante la aplicación de la prueba de Kaiser-Meyer-Olkin (KMO), con valores mayores a 0,5 , concretamente 0,827 , y la prueba de Esfericidad de Bartlett, con valores significativos $(\mathrm{sig}=0.000)$.

\section{b) Análisis factorial confirmatorio}

El análisis factorial estimado confirma la existencia de cuatro factores (subescalas). El ajuste de las hipótesis del modelo de cuatro factores $\left(X^{2}(896)=1,854.99, p=0,00\right)$ fue comparado con un modelo único de factor latente $\left(X^{2}(902)=2330,29, p=0,00\right)$. La comparación mostró un cambio estadísticamente significativo en la prueba Chi- cuadrado $\left(\Delta X^{2}=455, \Delta d f=6, p<0,05\right)$. Este cambio indica que el modelo de cuatro factores reproduce mejor la matriz de covarianzas observada que el modelo unidimensional.

\section{c) Revisión de la Escala de Justicia Social}

Tras los análisis realizados, los ítems que cargaban por debajo de 0,50 a cada factor (subescala) fueron eliminados. Además, se analiza con qué factor se relacionan los ítems. Así, se eliminaron aquellos ítems que cargaban más fuertemente ( $>7$ puntos) en un factor que no correspondía con el factor que la hipótesis sugería. El resultado combinado de estos análisis fue la eliminación de los ítems 27, 31, 33, 37, 38 y 42 en el factor 1 . En el factor 2 solamente fue eliminado el ítem 15. En el factor 3 se eliminó el ítem 36. El factor 4 no vio reducido su número de ítems.

\section{d) Análisis factoriales confirmatorios con el modelo revisado}

Tras el análisis factorial se revisaron los cuatro factores (29 ítems) a través de su aplicación a una segunda muestra compuesta por 262 sujetos. Los resultados mostraron una vez más un buen ajuste al modelo $\left(X^{2}=740.54\right.$, df $\left.=371, p=0,00 ; R M S E A=0,06\right)$. El RMSEA para el segundo análisis fue 0,09 , ligeramente por encima del valor 0,08 señalado por Browne y Cudeck (1993) como un buen ajuste. Los índices de ajuste del modelo revisado fueron más altos en este último análisis, en comparación con el modelo ajustado de la primera muestra. 


\section{e) Consistencia interna}

Se calcularon las Alfas de Cronbach en cada subescala de toda la muestra utilizando el último modelo revisado. Las alfas obtenidas son: las actitudes $\alpha=0,95$; las normas subjetivas $\alpha=0,82$, el control del comportamiento percibido $\alpha=0,84$, y las intenciones $\alpha=0,88$, lo que indica una fuerte consistencia interna a través de los cuatro factores. Las correlaciones entre la escala variaron entre 0,34 y 0,58 , lo que sugiere distintas subescalas, pero relacionadas.

\section{f) Validez convergente y discriminante}

Para evaluar la validez convergente y discriminante, las cuatro subescalas de justicia social se correlacionaron con las medidas obtenidas por las cuatro escalas de justicia social aplicadas (escalas de motivación por el servicio público, de creencia global en un mundo justo, de racismo simbólico y de neosexismo).

Tras los análisis de correlación de las diferentes escalas, los resultados obtenidos muestran que todas las subescalas de justicia social se correlacionaron positivamente con la motivación para participar en el servicio público y el apoyo a la validez convergente. Por otra parte, las subescalas se correlacionaron negativamente con neosexismo, el racismo simbólico y la creencia global en un mundo justo, apoyando así la validez discriminante de la SJS (Tabla 1).

\section{Procedimiento}

\section{Primera fase del estudio}

Para llevar a cabo el estudio de validación de la Escala de Justicia Social se realizó una traducción de los ítems de manera rigurosa, fiel y ajustada al contexto castellanohablante, con apoyo de expertos universitarios para las traducciones. El proceso de traducción duró alrededor de dos meses (entre enero y marzo de 2016) y estuvo compuesto por las tres fases que se muestran en la Figura 2. 
Figura 2. Fases de la traducción de la Escala de Justicia Social de Torres-Harding et al. (2012)

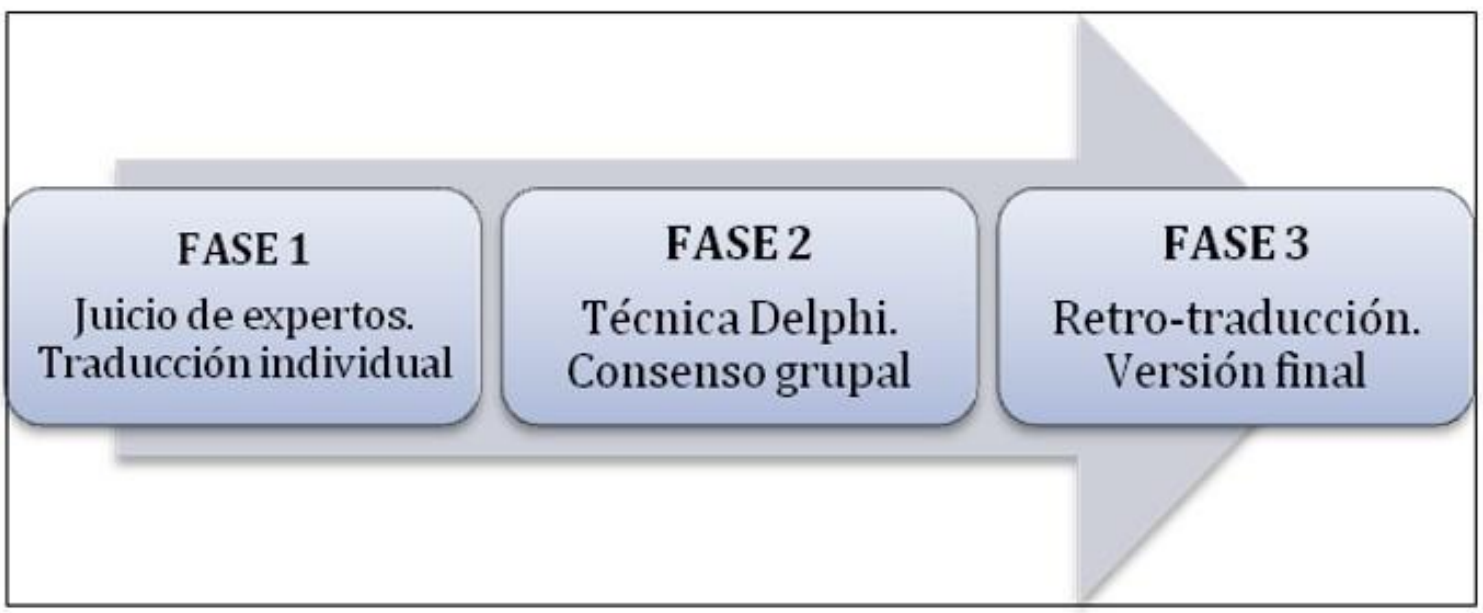

Fuente: Elaboración propia (2017)

Las fases que se siguieron para la traducción de la escala fueron las siguientes:

- Traducción individual por juicio de expertos. En primer lugar, se llevó cabo una traducción por 10 expertos. La traducción por juicio de expertos "consiste, básicamente, en solicitar a una serie de personas la demanda de un juicio hacia un objeto, un instrumento, un material de enseñanza, o su opinión respecto a un aspecto concreto" (Cabero y Llorente, 2013, p.14). Estos 10 expertos han sido elegidos por su competencia experta, tanto en el idioma (en este caso inglés) como en el objeto, es decir, la justicia social. Esta primera fase de traducción por juicio de expertos duró aproximadamente un mes y cuando todos los expertos tenían el instrumento traducido se llevó a cabo la segunda fase.

- Consenso grupal: Técnica Delphi. Esta técnica es un método de predicción sistemático interactivo a partir de una comunicación estructurada que se basa en un panel de expertos. Según Garrote y Del Carmen (2015), ofrece un gran nivel de interacción entre los expertos para llegar a un acuerdo o consenso, y brinda información esencialmente cualitativa, pero relativamente precisa, acerca del futuro. Una vez realizadas las traducciones individuales por juicio de expertos se llevó a cabo la técnica Delphi para llegar a un consenso grupal de la traducción de la Escala de justicia social. Se reunieron los 10 expertos y después de tres sesiones de discusión se consensuó una traducción final de la Escala de justicia social. 
- Retro-traducción (back-translation). Por último, se llevó a cabo una retrotraducción, entendida como el proceso de traducción de un documento que ya ha sido traducido a otra lengua de nuevo a la lengua original. Es necesario que esta retro-traducción sea realizada por un traductor experto independiente, para no estar sesgado por la primera traducción. Se eligió un nuevo traductor experto tanto en justicia social como en el dominio del inglés y se volvió a traducir la SJS a su lengua original, lo que permitió ver que dicha traducción era fiel al instrumento original.

Una vez realizada la traducción se pasó a realizar un análisis estadístico factorial para conocer la fiabilidad y la validez de la escala traducida.

\section{Segunda fase del estudio}

Con la versión traducida y validada se realizó un estudio ex post facto descriptivo.

La aplicación de la SJS traducida se hizo en mayo de 2016. La muestra total (Tabla 1) a la que se le suministró el cuestionario traducido fue seleccionada a través de un muestreo intencional.

La muestra final la conforman 326 sujetos de diferentes cursos del grado de Magisterio en Educación Infantil y en Educación Primaria que estudian en la Universidad Autónoma de Madrid correspondientes a 10 grupos de clase ( 5 grupos de cada grado). Concretamente el $54,6 \%$ de los estudiantes son de Educación Primaria y el $45,4 \%$ de Educación Infantil.

Del total de la muestra el $78,5 \%$ son mujeres y el $21,5 \%$ restante hombres, proporción análoga a la de estudiantes de estas especialidades. Las mujeres predominan especialmente en la muestra seleccionada de Infantil, circunstancia dada por las propias características del grado de Magisterio de Educación Infantil.

Tabla 1. Características de la muestra

\begin{tabular}{llcc}
\hline & & \multicolumn{2}{c}{ TITULACIÓN } \\
& & Educación primaria & Educación infantil \\
\hline \multirow{2}{*}{ GÉNERO } & Hombre & 62 & 8 \\
& Mujer & 116 & 140 \\
& Total & 178 & 148 \\
\hline
\end{tabular}

Fuente: Elaboración propia (2017) 
Una vez recogidos los datos se realizaron análisis descriptivos de las respuestas de los estudiantes y se compararon las medias de los sujetos a través de la Prueba $T$ de Student.

\section{RESULTADOS}

\section{Validación de la traducción de la Escala de Justicia social}

A continuación, se muestran los resultados de la validación de la traducción al castellano de la Escala de Justicia Social. Se detalla la fiabilidad obtenida, su validad de constructo y consistencia interna.

Fiabilidad

La fiabilidad de la prueba adaptada al castellano (24 ítems) es alta ( $\alpha=0,904)$. Este resultado es tan solo ligeramente inferior al obtenido en la escala sin revisar $(\alpha=0,93)$ que incluía los 44 ítems propuestos por los autores. El Alpha de Cronbach obtenido garantiza que la adaptación de la escala al castellano continúa midiendo de manera fiable el concepto de justicia social seguido por Torres-Harding et al. (2012).

\section{Validez de constructo}

Para estudiar la validez de constructo se llevó a cabo un análisis factorial. La Tabla 2 muestra la matriz de componentes rotados. En coherencia con los resultados obtenidos con la escala original, el análisis factorial detalla la existencia de cuatro subescalas: Actitudes hacia la justicia social, Control de comportamiento percibido, Intenciones de comportamiento y Normas subjetivas. Los ítems que conforman cada categoría son:

1) Actitudes hacia la justicia social, compuesto por los 11 primeros ítems.

2) Control del comportamiento percibido: 5 ítems, del 12 al 16.

3) Intenciones de comportamiento: 4 ítems, del 21 al 24.

4) Normas subjetivas: 4 ítems, del 17 al 20. 
Tabla 2. Matriz de componentes rotados de la Escala de Justicia Social traducida al castellano

\begin{tabular}{|c|c|c|c|c|}
\hline & 1 & 2 & 3 & 4 \\
\hline $\begin{array}{l}\text { T1-Creo que es importante asegurarse de que todos los individuos y grupos tengan la } \\
\text { oportunidad de hablar y ser escuchados, especialmente aquellos pertenecientes a grupos } \\
\text { tradicionalmente ignorados o marginados. }\end{array}$ & ,773 & ,018 & ,098 &,- 019 \\
\hline $\begin{array}{l}\text { T2-Creo que es importante que los individuos y grupos puedan exponer y describir sus } \\
\text { problemas, experiencias y metas con sus propios términos. }\end{array}$ & ,794 & ,004 &, 049 & ,052 \\
\hline $\begin{array}{l}\text { T3-Creo que es importante hablar con otros/as sobre los sistemas sociales de poder, privilegio } \\
\text { y opresión. }\end{array}$ & ,681 & ,130 & ,165 & ,103 \\
\hline $\begin{array}{l}\text { T4-Creo que es importante tratar de cambiar las condiciones sociales compartidas si estas } \\
\text { causan sufrimiento e impiden el bienestar individual. }\end{array}$ & ,695 & ,122 &, 023 & ,124 \\
\hline $\begin{array}{l}\text { T5-Creo que es importante ayudar a los individuos y grupos a conseguir los objetivos vitales } \\
\text { que han elegido. }\end{array}$ & ,684 & ,238 & ,193 & ,011 \\
\hline T6-Creo que es importante promover el bienestar físico y emocional de los individuos y grupos. & ,823 & 100 &, 120 & 029 \\
\hline $\begin{array}{l}\text { T7-Creo que es importante respetar y apreciar las identidades sociales diversas de las } \\
\text { personas. }\end{array}$ & ,741 &, 119 & ,204 & ,039 \\
\hline $\begin{array}{l}\text { T8-Creo que es importante permitir a los otros/as participar de manera significativa en las } \\
\text { decisiones que afectan a su vida. }\end{array}$ &, 510 & ,091 & ,197 & ,078 \\
\hline $\begin{array}{l}\text { T9-Creo que es importante apoyar a las asociaciones e instituciones que ayudan a los } \\
\text { individuos y grupos a alcanzar sus propias metas. }\end{array}$ & ,633 & 139 & ,272 & ,075 \\
\hline $\begin{array}{l}\text { T10-Creo que es importante promover una distribución justa y equitativa de poderes de } \\
\text { negociación, obligaciones y recursos en nuestra sociedad. }\end{array}$ & ,703 &, 141 & ,112 & ,068 \\
\hline T11-Creo que es importante actuar para la justicia social. & ,652 & ,152 & ,192 & 132 \\
\hline T12-Confío en que puedo tener un impacto positivo en la vida de otras personas. & ,306 & ,628 &,- 024 &,- 034 \\
\hline $\begin{array}{l}\text { T13-Tengo la certeza de que poseo la capacidad para trabajar con individuos y grupos de } \\
\text { forma que los empodere. }\end{array}$ & ,094 & ,753 & ,109 & ,055 \\
\hline $\begin{array}{l}\text { T14-Si me decido a hacerlo, soy capaz de influir en otros/as para promover la justicia y la } \\
\text { igualdad. }\end{array}$ & ,065 & ,843 & ,203 & ,097 \\
\hline $\begin{array}{l}\text { T15-Confío en mi capacidad para hablar con otros/as acerca de las injusticias sociales y el } \\
\text { impacto de las condiciones sociales sobre la salud y el bienestar. }\end{array}$ & ,145 & ,716 & ,206 & ,152 \\
\hline T16-Tengo la certeza de que si lo intento puedo tener un impacto positivo en mi comunidad. &, 146 & ,729 & ,239 & 125 \\
\hline $\begin{array}{l}\text { T17-Las personas que me rodean están comprometidas en actividades que abordan las } \\
\text { injusticias sociales. }\end{array}$ &,- 035 & ,219 & ,102 & ,692 \\
\hline $\begin{array}{l}\text { T18-Las personas que me rodean sienten que es importante comprometerse con el diálogo } \\
\text { acerca de las injusticias sociales. }\end{array}$ &, 040 &, 044 & ,161 & ,841 \\
\hline T19-Las personas que me rodean apoyan los esfuerzos que promueven la justicia social. & ,122 & ,108 &, 120 & ,862 \\
\hline $\begin{array}{l}\text { T20-Las personas que me rodean son conscientes de las injusticias sociales y las } \\
\text { desigualdades de poder en nuestra sociedad. }\end{array}$ & ,191 &,- 026 & ,137 & ,577 \\
\hline $\begin{array}{l}\text { T21-En el futuro, haré todo lo que pueda por asegurarme de que todos los individuos y grupos } \\
\text { tengan la oportunidad de hablar y ser escuchados. }\end{array}$ & ,298 & ,139 & ,714 & 220 \\
\hline $\begin{array}{l}\text { T22-En el futuro, tengo la intención de hablar con otros/as sobre las injusticias sociales, las } \\
\text { desigualdades de poder y el impacto de las fuerzas sociales sobre la salud y el bienestar. }\end{array}$ & ,241 & ,169 & ,790 & ,234 \\
\hline $\begin{array}{l}\text { T23-En el futuro, tengo la intención de comprometerme en actividades que promuevan la } \\
\text { justicia social. }\end{array}$ & ,291 & ,299 &, 774 & 191 \\
\hline $\begin{array}{l}\text { T24-En el futuro, tengo la intención de trabajar colaborativamente con otros/as para que } \\
\text { puedan definir sus problemas y desarrollar la capacidad para resolverlos. }\end{array}$ & ,221 & ,209 & ,797 & ,091 \\
\hline
\end{tabular}

Fuente: Elaboración propia (2017) 
Los porcentajes de varianza explicados aportan información de la cantidad de varianza total explicada por cada factor, así como el porcentaje de varianza explicada total y acumulada. En este caso, como se ha detallado, se extraen cuatro factores que explican el $60,36 \%$ de la varianza total (Tabla 3 ).

Tabla 3. Varianza explicada por cada factor (suma de las saturaciones al cuadrado de la rotación)

\begin{tabular}{lccc}
\hline & TOTAL T & \% VARIANZA & \% ACUMULADO \\
\hline 1. Actitudes hacia la justicia social & 5,932 & 24,716 & 24,716 \\
2. Control percibido de comportamiento & 3,142 & 13,092 & 37,808 \\
3. Intenciones de comportamiento & 2,885 & 12,021 & 49,830 \\
4. Normas subjetivas & 2,528 & 10,535 & 60,365 \\
\hline
\end{tabular}

Fuente: Elaboración propia (2017)

\section{Consistencia interna}

La fiabilidad de cada subescala para toda la muestra permite comparar la consistencia interna de las consiguientes subescalas de la versión original y traducida. Los valores del Alfa de Cronbach obtenidos son similares a los de la escala original (Tabla 4, incluye ejemplos de ítems que más cargan en cada subescala según los resultados obtenidos del análisis factorial).

Tabla 4. Subescalas de la Escala de Justicia Social, ejemplos de ítems y coeficientes de consistencia interna

\begin{tabular}{|c|c|c|c|}
\hline \multirow{2}{*}{$\begin{array}{c}\text { Subescalas } \\
\text { (con número de items) }\end{array}$} & \multirow{2}{*}{ Ejemplo de items } & \multicolumn{2}{|c|}{ Consistencia Interna (Alfa de Cronbach) } \\
\hline & & Escala traducida & Escala original revisada \\
\hline $\begin{array}{l}\text { 1. Actitudes hacia la } \\
\text { justicia social (11) }\end{array}$ & $\begin{array}{l}\text { T6. Creo que es importante } \\
\text { promover el bienestar físico y } \\
\text { emocional de los individuos y grupos }\end{array}$ & 0,908 & 0,95 \\
\hline $\begin{array}{l}\text { 2. Control de } \\
\text { comportamiento } \\
\text { percibido (5) }\end{array}$ & $\begin{array}{l}\text { T14. Si me decido a hacerlo, soy } \\
\text { capaz de influir en otros/as para } \\
\text { promover la justicia y la igualdad }\end{array}$ & 0,83 & 0,84 \\
\hline $\begin{array}{l}\text { 3. Intenciones de } \\
\text { comportamiento (4) }\end{array}$ & $\begin{array}{l}\text { T24. En el futuro, tengo la intención } \\
\text { de trabajar colaborativamente con } \\
\text { otros/as para que puedan definir sus } \\
\text { problemas y desarrollar la capacidad } \\
\text { para resolverlos }\end{array}$ & 0,885 & 0,88 \\
\hline 4. Normas subjetivas (4) & $\begin{array}{l}\text { T19. Las personas que me rodean } \\
\text { apoyan los esfuerzos que } \\
\text { promueven la justicia social }\end{array}$ & 0,766 & 0,82 \\
\hline
\end{tabular}

Fuente: Elaboración propia (2017) 
La fiabilidad obtenida para cada subescala es: las actitudesa $=0,90$; las normas subjetivas $\mathrm{\alpha}=0,88$, el control percibido del comportamiento $\alpha=0,83, \quad y$ las intencionesa $=0,76$. Estos valores indican una fuerte consistencia interna de las cuatro subescalas de la misma manera que sucede en la escala original revisada. Las correlaciones entre las escalas confirman la distinción entre cuatro de las subescalas (Tabla 5).

Tabla 5. Correlación de Pearson entre las subescalas de justicia social

\begin{tabular}{llccc}
\hline & & $\mathbf{2}$ & $\mathbf{3}$ & $\mathbf{4}$ \\
\hline \multirow{2}{*}{ 1. Actitudes hacia la justicia social } & $p$ & 0,00 & 0,00 & 0,00 \\
& Sig. (bilat) & 1,00 & 1,00 & 1,00 \\
\hline \multirow{2}{*}{ 2. Control de comportamiento percibido } & $p$ & - & 0,00 & 0,00 \\
& Sig. (bilat) & & 1,00 & 1,00 \\
\hline \multirow{2}{*}{ 3. Intenciones de comportamiento } & $p$ & - & - & 0,00 \\
& Sig. (bilat) & & & 1,00 \\
\hline
\end{tabular}

Fuente: Elaboración propia (2017)

\section{Estudio descriptivo de las actitudes y del compromiso que los futuros docentes tienen acerca de la justicia social}

En este apartado se da respuesta al segundo objetivo de la investigación. Se aplicó la Escala de Justicia Social traducida para conocer con mayor profundidad cómo se comporta la muestra seleccionada y confirmar a un $95 \%$ de seguridad si las diferencias encontradas son aplicables a toda la población.

Los resultados de los análisis descriptivos de la escala traducida en función de la titulación de grado de magisterio de la muestra y en función de su género aparecen en las Tablas 6 y 7 . Los datos apuntan a lo siguiente:

- Los futuros maestros de Educación Infantil cuentan con mayores actitudes hacia la justicia social que los maestros de Primaria (una diferencia de 0,39 puntos más). Por otro lado, son las mujeres las que más actitudes hacia la justicia social tienen, 0,14 puntos las mujeres frente al $-0,52$ puntos los hombres (ítems T1 a T11).

- El control de comportamiento percibido es mayor en los futuros maestros de Educación Primaria ( 0,15 puntos) frente a la puntuación negativa de los futuros maestros de Educación Infantil (-0,18 puntos). Son los hombres los que más puntúan en esta subescala de Justicia Social ( 0,39 puntos) frente al bajo nivel de control del comportamiento percibido por las mujeres $(-0,11$ puntos) (ítems T12 a T16). 
- Los futuros maestros de Educación Primaria cuentan con una ligera mayor intención de comportamiento hacia la justicia que quienes cursan Educación Infantil ( 0,05 puntos más). Esta diferencia aumenta en función del género, las mujeres tienen 0,21 puntos más de intención por comportarse hacia la justicia social que los hombres (ítems T17 a T20).

- Los futuros maestros de Educación Infantil creen que hay mayor cantidad de normas subjetivas en relación con la justicia social que sus compañeros de Educación Primaria $(0,94$ y $\quad-0,07$ respectivamente). Esta diferencia prácticamente se anula en función del género, donde los hombres y las mujeres usualmente no sienten la existencia de normas (ítems T21 a T24).

Tabla 6. Puntuaciones medias y desviación típica en los factores en función de la titulación

\begin{tabular}{lcccc}
\hline & \multicolumn{2}{c}{ EducAción PRIMARIA } & \multicolumn{2}{c}{ EducAción INFANTIL } \\
& Media & Desv. típica & Media & Desv. típica \\
\hline 1. Actitudes hacia la justicia social & $-0,179$ & 1,089 & 0,215 & 0,836 \\
2. Control de comportamiento percibido & 0,151 & 0,927 & $-0,181$ & 1,056 \\
3. Intenciones de comportamiento & 0,023 & 1,003 & $-0,027$ & 1,000 \\
4. Normas subjetivas & $-0,078$ & 1,041 & 0,093 & 0,943 \\
\hline
\end{tabular}

Fuente: Elaboración propia (2017)

Tabla 7. Puntuaciones medias y desviación típica en los factores en función del género

\begin{tabular}{lcccc}
\hline & \multicolumn{2}{c}{ HOMBRE } & \multicolumn{2}{c}{ MUJER } \\
& Media & Desv. típica & Media & Desv. típica \\
\hline 1. Actitudes hacia la justicia social & $-0,517$ & 1,363 & 0,143 & 0,821 \\
2. Control de comportamiento percibido & 0,386 & 0,910 & $-0,107$ & 0,999 \\
3. Intenciones de comportamiento & $-0,167$ & 1,034 & 0,046 & 0,988 \\
4. Normas subjetivas & 0,007 & 0,911 & $-0,002$ & 1,025 \\
\hline
\end{tabular}

Fuente: Elaboración propia (2017)

Más allá de las diferencias encontradas para la muestra en torno a la titulación y al género, la prueba T-Student permite estudiar si dichas diferencias son significativas para toda la población asumiendo un error del 5\%. Las tablas 8 y 9 detallan los resultados de la prueba de Levene para saber si las varianzas de ambos grupos (Educación PrimariaEducación Infantil y Hombre-Mujer) son homogéneas y la de T-Student para conocer si, efectivamente, se pueden elaborar teorías diferenciales para toda la población en función de cada variable de agrupación. 
Tabla 8. Prueba T-Student de las subescalas de justicia social en función de la titulación

\begin{tabular}{lccccc}
\hline & \multicolumn{2}{c}{ Prueba de LeVene } & \multicolumn{3}{c}{ Prueba T-S tudent } \\
& $\mathrm{F}$ & Sig. & $\mathrm{t}$ & $\mathrm{gl}$ & Sig. (bilat) \\
\hline 1. Actitudes hacia la justicia social & 8,46 & 0,00 & $-3,68$ & 318,84 & 0,00 \\
2. Control de comportamiento percibido & 3,35 & 0,07 & 3,00 & 321,00 & 0,00 \\
3. Intenciones de comportamiento & 0,21 & 0,65 & 0,44 & 321,00 & 0,66 \\
4. Normas subjetivas & 0,72 & 0,40 & $-1,53$ & 321,00 & 0,13 \\
\hline
\end{tabular}

Fuente: Elaboración propia (2017)

Los valores de estadístico $\mathrm{F}$ de la prueba de Levene señalan que las varianzas de los grupos Educación Primaria e Educación Infantil son homogéneas para las subescalas Intenciones de comportamiento $(\mathrm{F}=0,21)$ y Normas subjetivas $(\mathrm{F}=0,72)$. Por su parte, los resultados ofrecidos por la prueba T-Student muestran que, efectivamente, existen diferencias significativas en función de la titulación que cursan los futuros maestros en relación a las Actitudes hacia la justicia social y el Control del comportamiento percibido.

Tabla 9. Prueba T-Student de las subescalas de justicia social en función del género

\begin{tabular}{lccccc}
\hline & \multicolumn{2}{c}{ PRUeba de LeVENe } & \multicolumn{3}{c}{ PRUEba T-S TUdent } \\
& $\mathrm{F}$ & Sig. & $\mathrm{t}$ & $\mathrm{gl}$ & Sig. (bilat) \\
\hline 1. Actitudes hacia la justicia social & 30,57 & 0,00 & $-3,86$ & 83,32 & 0,00 \\
2. Control de comportamiento percibido & 4,11 & 0,04 & 3,92 & 119,09 & 0,00 \\
3. Intenciones de comportamiento & 0,88 & 0,34 & $-1,58$ & 321 & 0,11 \\
4. Normas subjetivas & 1,61 & 0,20 & 0,06 & 321 & 0,94 \\
\hline
\end{tabular}

Fuente: Elaboración propia (2017)

De igual manera, son las dos primeras subescalas de justicia social, Actitudes hacia la justicia social y Control del comportamiento percibido, las que cuentan con una puntuación diferente en función del género del futuro maestro ( $t=-3,86 \alpha=0,00$ y $t=3,92$ $\alpha=0,00$, respectivamente). No parece haber diferencias significativas en la opinión de hombres y mujeres en torno a las subescalas Intenciones de comportamiento y Normas subjetivas. El estadístico $\mathrm{F}$ apunta a que las varianzas de los grupos correspondientes a hombres y mujeres son homogéneas para estas dos últimas subescalas (Intenciones de comportamiento: $F=0,88$; Normas subjetivas: $F=1,61$ ). 


\section{CONCLUSIONES Y DISCUSIÓN}

Los análisis reflejan una importante coherencia respecto a los resultados de la escala original de Torres-Harding et al. (2012), por lo que se confirma la correcta traducción de los ítems al castellano. Esta concordancia permite afirmar que la traducción propuesta en este trabajo constituye una valiosa herramienta para los investigadores castellanohablantes que busquen identificar valores, comportamientos y actitudes relacionados con la justicia social. La validación a través de una modesta muestra puede ser considerada una limitación del estudio. Sin embargo, la coherencia total encontrada en los resultados con respecto a la escala original señala que los análisis han sido suficientes para confirmar la importancia de tener una escala que mida esas actitudes en español. El hecho de que la muestra estuviera compuesta por futuros maestros es un elemento a destacar. Este estudio aporta algunos indicios que relacionan las actitudes de los maestros hacia la justicia social y la implicación con su futura práctica docente.

Respecto a los resultados obtenidos con los estudiantes de magisterio, se vio que existen diferencias en cuanto a la titulación y al género en las diferentes subescalas de justicia social, lo que abre nuevas posibilidades de estudio que profundicen en la comprensión de dichas discrepancias. Un ejemplo puede ser un estudio cualitativo que aborde las percepciones y significaciones de estos futuros profesionales, así como estudios complementarios con docentes en ejercicio. El concepto de justicia social implícito en esta escala se articula a través de la redistribución, que es una buena aproximación a la construcción de una herramienta que ayude a identificar acciones hacia la justicia social. Asimismo, algunos otros ítems hacen referencia al reconocimiento y a la participación, dimensiones fundamentales para lograr una sociedad más justa. No obstante, sería interesante que la escala fuese revisada e incluyese un número de ítems equitativo de cada una de las tres dimensiones.

El presente estudio aporta una herramienta en español, así como datos sobre un tema de alta relevancia educativa y social. Hasta el momento no se disponía de ninguna escala para medir las actitudes de las personas hacia la justicia social en el contexto iberoamericano, motivo que permite seguir avanzando en esta temática en un contexto tan necesario como España y América Latina. La comparación de los hallazgos de la presente investigación con los encontrados por el artículo original de Torres-Harding (2012) vislumbran ciertas diferencias en las actitudes hacia la justicia social, motivo por el cual sería necesario seguir profundizando en los motivos de estas diferencias. Si se quiere cambiar la sociedad hay que empezar por las escuelas, y las actitudes que los docentes y futuros docentes tengan sobre la justicia social juega un papel fundamental. En este sentido, solo si los docentes son conscientes de sus actitudes serán capaces de entender mejor sus acciones y mejorarlas para lograr una mayor justicia en las aulas y en la sociedad. Si los docentes están comprometidos con la lucha contra las injusticias, tienen en sus manos el poder de convertir la educación - y con ella, la sociedad-en un lugar más justo, crítico y democrático. 


\section{REFERENCIAS}

Adams, M., Bell, L. A., y Griffin, P. (Eds.). (2007). Teaching for diversity and social justice. Nueva York, NY: Routledge.

Ajzen, I. (1991). The theory of planned behavior. Organizational Behavior and Human Decision Processes, 50(2), 179-211.

Browne, M., y Cudeck, R. (1993). Alternative ways of assessing model fit. En K. Bollen y S. Long (Eds.), Testing structural equation models (pp. 136-162). Newbury Park, CA: Sage.

Cabero Almenara, J., y Llorente Cejudo, M. C. (2013), La aplicación del juicio de experto como técnica de evaluación de las tecnologías de la información (TIC). Revista de Tecnología de Información y Comunicación en Educación, 7(2),11-22.

Caprara, G., Vecchione, M., Capanna, C., y Mebane, M. (2009). Perceived political self-efficacy: Theory, assessment, and applications. European Journal of Social Psychology, 39(6), 1002-1020.

Colquitt, J. (2001). On the dimensionality of organizational justice: A construct validation of a measure. Journal of Applied Psychology, 86(3), 386-400.

Constantine, M., Hage, S., Kindaichi, M., y Bryant, R. (2007). Social justice and multicultural issues: Implications for the practice and training of counselors and counseling psychologists. Journal of Counseling and Development, 85(1), 24-29.

Cook, S. (1990). Toward a psychology of improving justice: Research on extending the equality principle to victims of social injustice. Journal of Social Issues, 46(1), 147-161.

Corning, A., y Myers, D. (2002). Individual orientation towards engagement in social action. Political Psychology, 23(4), 703-729.

Coursey, D., y Pandey, S. (2007). Public service motivation measurement: Testing an abridged version of Perry's proposed scale. Administration and Society, 39(5), 547-568.

Dean, J. K. (2009). Quantifying social justice advocacy competency: Development of the social justice advocacy scale (Tesis doctoral). Georgia State University, Atlanta, GA.

Fabian, C. G. (2012). Social Identity and Social Justice Orientation among Social Work Graduate Students: Examining the Role of Perceived Injustice and Self-Efficacy (Tesis doctoral). University of Michigan, Ann Arbour, Ml.

Fietzer, A. W., y Ponterotto, J. (2015). A Psychometric Review of Instruments for Social Justice and Advocacy Attitudes. Journal for Social Action in Counseling and Psychology, 7(1), 19-40.

Fouad, N., Gerstein, L., y Toporek, R. (2006). Social justice and counseling psychology in context. En R. Toporek, L. Gerstein, N. Fouad, G. Roysircar y T. Israel (Eds.), Handbook for social justice in counseling psychology: Leadership, vision, and action (pp. 1-15). Thousand Oaks, CA: Sage.

Fox, D. (2003). Awareness is good, but action is better. The Counseling Psychologist, 31(3), 299-304.

Fraser, N. (2006). Reinventar la justiciaen un mundo globalizado. New Left Review, 36(2), 34-53. 
Fraser, N. (2008). Scales of Justice: Reimagining Political Space in a Globalizing World. Nueva York, NY: Columbia University Press.

Fraser, N., y Honneth, A. (2006). ¿Redistribución o reconocimiento?: un debate filosófico-político. Madrid, España: Morata.

Garrote, P. R., y Del Carmen, M. (2015), La validación por juicio de expertos: dos investigaciones cualitativas en Lingüística aplicada. Revista de lingüística, 18. Recuperado de http://www.nebrija.com/revista-linguistica/la-validacion-por-juicio-de-expertos-dosinvestigaciones-cualitativas-en-linguistica-aplicada

Goodman, D. (2000). Motivating people from privileged groups to support social justice. Teachers College Record, 102(6), 1061-1085.

Goodman, L.A., Liang, B., Helms, J., Latta, R.E, Sparks, E., y Weintraub, S. (2004). Training counseling psychologists as social justice agents: Feminist and multicultural principles in action. The Counseling Psychologist, 32(6), 793-837.

Griffiths, M. (2003). Action for Social Justice in Education. Maidenhead, Inglaterra: Open University Press. Henry, P., y Sears, D. (2002). The symbolic racism 2000 scale. Political Psychology, 23(2), 253-283.

Honneth, A. (2007). Reificación: un estudio en la teoría del reconocimiento. Madrid, España: Katz.

Kozlowski, C., Ferrari, J., y Odahl, C. (2014). Social Justice and Faith Maturity: Exploring Whether Religious Beliefs Impact Civic Engagement. Education, 134(4), 427-432.

Lipkus, I. (1991). The construction and preliminary validation of a global belief in a just world scale and the exploratory analysis of the multidimensional belief in a just world scale. Personality and Individual Differences, 12(11), 1171-1178.

Marías, J. (1974). La justicia social y otras justicias. Madrid, España: Seminarios y Ediciones.

Martínez-Garrido, C., y Perines Véliz, H. (2015). Estudio de las actitudes de los futuros docentes sobrejusticia social. En AIDIPE (Ed.), Investigar con y para la sociedad. Vol. 1 (pp. 349-357). Cádiz, España: Bubok.

Murillo, F. J., y Hernández-Castilla, R. (2011a). Hacia un concepto de justicia social. REICE. Revista Iberoamericana sobre Calidad, Eficacia y Cambio en Educación, 9(4), 7-23.

Murillo, F. J., y Hernández-Castilla, R. (2011b). Trabajar por la justicia social desde la Educación. Editorial. REICE. Revista Electrónica Iberoamericana sobre Calidad, Eficacia y Cambio en Educación, 9(4), 3-6.

Perry, J. (1996). Measuring public service motivation: An assessment of construct reliability and validity. Journal of Public Administration Research and Theory, 6(1), 5-22.

Poteat, V. P., Scheer, J. R., Marx, R. A., Calzo, J. P., y Yoshikawa, H. (2015). Gay-Straight Alliances Vary on Dimensions of Youth Socializing and Advocacy: Factors Accounting for Individual and SettingLevel Differences. American journal of community psychology, 55(3-4), 422-432.

Prilleltensky, I., y Nelson, G. (2002). Doing psychology critically: Making a difference in diverse settings. Houndmill, Inglaterra: Palgrave MacMillan. 
Prilleltensky, I. (2001). Value-based praxis in community psychology: Moving towards social justice and social action. American Journal of Community Psychology, 29(5), 747-778.

Rasinski, K. (1987). What's fair is fair-or is it? Value differences underlying public views about social justice. Journal of Personality and Social Psychology, 53(1), 201-211.

Ritchhart, R. (2002). Development of the social activism beliefs rating scale (SABR): An instrument to measure psychologists' attitudes toward social activism. Dissertation abstracts international: Section B: The Sciences and Engineering, 63(6-B), 30-70.

Rawls, J. (1971). A theory of justice. Cambridge, MA: Harvard University Press.

Toporek, R., y Williams, R. (2006). Ethics and professional issues related to the practice of social justice in counseling psychology. En R. Toporek, L. Gerstein, N. Fouad, G. Roysircar y T. Israel (Eds.), Handbook for social justice in counseling psychology: Leadership, vision, and action (pp. 17-34). Thousand Oaks, CA: Sage.

Torres-Harding, S., Siers, B., y Olson, B. (2012). Development and Psychometric evaluation of the Social Justice Scale (SJS). American Journal Community Psychology, 50(1-2), 77-88.

Torres-Harding, S., Carollo, O., Schamberger, A., y Clifton-Soderstrom, K. (2013). Values and Religiosity as Predictors of Engagement in Social Justice. Journal of Prevention \& Intervention in the Community, 41(4), 255-266.

Torres-Harding, S., Diaz, E., Schamberger, A., y Carollo, O. (2015). Psychological Sense of Community and University Mission as Predictors of Student Social Justice Engagement. Journal of Higher Education Outreach and Engagement, 19(3), 89-112.

Tougas, F., Brown, R., Beaton, A., y Stéphane, J. (1995). Neosexism: Plus ça change, plus c'est pareil. Personality and Social Psychology Bulletin, 21, 842-849.

Young, I. M. (2007). Global Challenges: War, Self-Determination, and Responsibility for Justice. Londres, Inglaterra: Oxford Polity Press.

Young, I. M. (2000). Inclusion and Democracy. Oxford, Inglaterra: Oxford University Press.

Young, I. M. (2011). Responsibility for Justice. Londres, Inglaterra: Oxford Polity Press. 